\title{
Relación entre Productividad e Ingresos en el Sector Macanero del Cantón Gualaceo, Provincia del Azuay
}

\section{Relationship between Productivity and Income in the Macanero Sector of the Canton of Gualaceo, Province of Azuay}

\author{
Gabriela Amoroso Castro $^{1 *}$, María Elena Calle Calle ${ }^{1}$ y Mónica Rosales Namicela ${ }^{1}$ \\ ${ }^{1}$ Universidad Católica de Cuenca \\ *gamorosoc@ucacue.edu.ec
}

DOI: https://doi.org/10.26871/killkana_social.v2i3.345

\begin{abstract}
Resumen
Esta investigación se realizó dando continuidad a un análisis, en el que se relacionaron las variables de ingresos y canales de comercialización de los artesanos macaneros; al considerar que los ingresos que perciben, no corresponden al esfuerzo, tiempos de dedicación, valor agregado, transferencia del conocimiento ancestral intergeneracional, entre otros; se abordó la temática con el objetivo, de analizar la relación entre la productividad y los ingresos que genera la actividad artesanal del tejido de macanas de las parroquias de Bullcay y Bullzhún del cantón Gualaceo; ubicado en la provincia del Azuay - Ecuador. Se enmarca dentro de la línea de investigación de la economía social y solidaria, con enfoque cuantitativo de tipo no experimental - transversal y descriptivo, porque analiza la relación entre las variables de productividad e ingresos asociados a la edad de los 36 artesanos macaneros considerados como universo de estudio y el tiempo de dedicación a la actividad. Entre los principales resultados se pudo apreciar que el índice de correlación de Pearson entre la edad de los artesanos y la cantidad de unidades producidas es muy débil al ser $\rho=-0,182$. El índice de productividad es de 0,043 $\mathrm{ud}$./h, lo que equivale a la producción de 7 macanas por mes que genera un ingreso mensual de $238 \mathrm{USD}$, valor inferior al de un salario básico unificado. El índice de productividad obtenido refleja la realidad del sector que desarrolla una actividad artesanal manual que demanda mayor tiempo de dedicación y no existe el reconocimiento al valor intangible como conocimiento y cultura, en términos monetarios en el mercado.
\end{abstract}

Palabras clave: productividad, ingresos, edad, artesanos macaneros.

\begin{abstract}
This research was conducted, giving continuity to an analysis, in which related variables income and marketing channels of macaneros craftsmen; considering that the revenue that perceive, does not correspond to the effort, time of dedication, added value, transfer of intergenerational, ancestral knowledge among others. addressed the issue with the aim of analyzing the relationship between productivity and revenues generated by the craft of the tissue of the parishes of Bullcay and Bullzhún of the canton Gualaceo macanas; It is located in the province of Guayas - Ecuador. Is part of the research line of the social economy and solidarity, with non-experimental quantitative approach - cross-sectional and descriptive, because it analyzes the relationship between the variables of productivity and revenue associated with the age of 36 macaneros craftsmen considered as universe of study and dedication to the activity time. Among the main results demonstrated that the index of Pearson correlation between the age of the craftsmen and the number of units produced is very weak as $\rho=-0,182$. The productivity index is $0.043 \mathrm{ud} / \mathrm{h}$, equivalent to the production of 7 clubs per month that generates a monthly income of 238 USD, less than a unified basic salary. The productivity index retrieved reflects the reality of the sector that develops a manual craft activity that demands more time dedication and there is recognition of the value of intangible knowledge and culture, in monetary terms in the market.
\end{abstract}

Key words: productivity, income, age, macaneros craftsmen.

\section{Introducción}

El Ecuador al ser un país diverso, multiétnico y multicultural, alberga contenido inmaterial reflejado en la elaboración de las artesanías en las diferentes regiones; en las parroquias de Bullcay y Bullzhún, ubicadas en el cantón Gualaceo, provincia del Azuay; la mayoría de sus habi- tantes se dedican a la actividad artesanal textil, elaborando macanas y productos aplicando la técnica Ikat.

Es de interés de las autoras, determinar de qué manera los artesanos pueden mejorar sus ingresos provenientes de la actividad, reconociendo un justo valor al trabajo realizado, de forma que ésta pueda convertirse en su principal 
fuente de ingresos y dedicación, identificando las variables de mayor incidencia; para ello se ha iniciado, con una serie de análisis correlacionales, en los cuales se relacionan diferentes variables con los ingresos; considerando que en este sector, más del $80 \%$ de sus habitantes se dedican a la producción de macanas y otros artículos o prendas de vestir con la técnica ancestral del IKAT; esta actividad que a más de ser un referente histórico de identidad propia del sector; ha sido declarado con un Patrimonio Inmaterial de la Humanidad por la UNESCO.

Para el caso de los artesanos los ingresos son aquellos valores monetarios que perciben por la venta de sus productos y/o actividades complementarias que se sienten obligados a realizar para redondear sus ingresos y así cubrir sus necesidades básicas; (Mordo, 2002) destaca la dura situación que atraviesa el sector artesanal Latinoamericano al exponer que la gran mayoría de estos grupos mantienen una condición de pobreza esto quiere decir que los ingresos percibidos por su actividad es bajo y no les permite satisfacer sus necesidades elementales; por esta situación, la gran mayoría de artesanos se ven abocados a insertarse en el mercado laboral formal o informal, y en actividades agrícolas, entre otras.

Mediante un estudio de las condiciones socioeconómicas de los artesanos se pudo concluir que mantienen una situación de pobreza; al ser una actividad $100 \%$ artesanal demanda muchas horas de dedicación, pero sin un reconocimiento monetario adecuado por parte del mercado, así el $52,8 \%$ de los artesanos perciben ingresos menores a un Salario Mínimo Vital General (SMVG) de 386,00 USD, un $36 \%$ perciben menos de $\$ 600,00$ USD y sólo un $11 \%$ perciben ingresos sobre los 600,00 USD mensuales; asî también se destaca que el nivel de productividad es bajo, más del $80 \%$ de los artesanos producen alrededor de 1 a 15 macanas por mes, otro hecho a destacar es el costo de producción que oscila entre 19 y 35 dólares. Además, en dicho estudio se menciona que más del $70 \%$ de los artesanos destina menos de 280,00 dólares mensuales de sus ingresos para atender sus necesidades básicas, lo que quiere decir que, ni siquiera pueden cubrir las necesidades básicas con los ingresos que perciben porque además tiene que cubrir sus costos de producción, esto complica aún más la situación porque, no pueden desarrollar una capacidad de ahorro y por lo tanto no pueden acceder a procesos de financiamiento para mejorar su productividad y generar procesos de innovación.

Finalmente, de un estudio desarrollado por la FLACSO respecto a la situación de los artesanos ecuatorianos se reafirma la evidencia que una de las amenazas que atraviesa el sector artesanal es la "Poca valoración artesanal" (FLACSO, 2010), a más de otros factores que afectan gravemente la condición de los artesanos y la supervivencia de la actividad.

Al observar como una realidad, que la remuneración que perciben los artesanos mayoritariamente se encuentra subvalorada y no corresponden al esfuerzo, tiempos de dedicación y valor agregado, desde el traslado del conocimiento y cultura ancestral intergeneracional, otros autores han servido de soporte en el análisis de la productividad, tales como Kotler 2009, Smith 2002, Taylor 1973, Sumanth 1999, Prokopenko 1989; de igual manera, Valle 1991 analiza la productividad con visión marxista, Solow 1957, habla de productividad multifactorial; mientras que con relación a los factores determinantes de la productividad y competitividad de empresas locales, se tiene a Alburquerque 1999, Cordero y otros 2003, Díaz Sánchez y Guadarrama 2006, Medina 2009, Parkin y Loría 2010 y Vázquez y Baquero 2005.

Para el desarrollo de la presente investigación se ha considerado la propuesta del modelo de productividad parcial dada por Sumanth, 1990, que coincide con la propuesta de la Organización para la Cooperación y Desarrollo Económico (OCDE) 1950, considerando que el capital humano es un factor fundamental del sector artesanal y dentro de él, la experticia y experiencia. La productividad e ingresos con base en la teoría de ventaja competitiva de David Ricardo, analizada por Ríos 2018, han sido también instrumentos considerados para el desarrollo del marco teórico del presente trabajo y, los datos publicados en una investigación previa de las autoras, que relacionaron los ingresos con los canales de comercialización; en donde se evidenció que los artesanos del sector, registran ingresos inferiores a un SMVG, sugiriendo "realizar nuevos estudios que asocien los ingresos con variables más determinantes tales como innovación, productividad, asociatividad, estrategias de marketing, entre otros" (Amoroso, Calle, Campoverde, y Rosales, 2018)

Con base en lo mencionado, en esta ocasión se analizará la relación entre la productividad y los ingresos de la actividad artesanal del tejido de macanas, asociada a la edad de los artesanos, considerando información primaria recopilada a través del proyecto de vinculación ejecutado en el 2016 por la carrera de Economía de la Universidad Católica de Cuenca, en convenio con el Gobierno Autónomo Descentralizado (GAD) cantonal de Gualaceo; para ello, a lo largo del documento se persiguen los siguientes objetivos; primero, fundamentar teóricamente los factores de productividad que inciden en los ingresos del sector artesanal; segundo, relacionar productividad con ingresos y edad mediante análisis correlacionales y descriptivo; finalmente calcular el índice de productividad parcial del sector. Con ello en esta investigación se busca dar respuesta a las interrogantes: ¿Cuál es la relación entre la productividad y los ingresos generados en el sector artesanal de macanas, asociados a la edad de los artesanos? ¿Cuál es el índice de productividad parcial considerando una jornada laboral legalmente establecida en el Ecuador?

Se asume que la productividad en el sector macanero se ve influenciada por la edad de los artesanos y el tiempo de dedicación a la actividad, lo que se refleja en los ingresos que perciben por la venta de los productos que elaboran; por lo tanto, las personas más jóvenes están en capacidad 
de elaborar más unidades de producto y por ende tener mayores ingresos por la actividad; es decir, la relación entre productividad e ingresos, asociados a la edad de las personas es directa.

Los resultados que aquí se generan serán de utilidad para el sector artesanal macanero como caso de estudio y para la comunidad en general, porque permitirá aclarar cuál es el panorama en el que se desenvuelven con relación a sus niveles de productividad y la capacidad para la generación de ingresos. Permitirá conocer los factores que afectan la productividad y bajo esa premisa inducir a los que necesitan fortalecimiento para mejorar dichos indicadores; información que puede ser considerada por el GAD cantonal de Gualaceo y el gobierno en general para el diseño e implementación de políticas que provean herramientas adecuadas para el mejoramiento de las condiciones de vida y producción del sector, proceso que puede ser acompañado conjuntamente con la academia desde su eje transversal de vinculación con la comunidad.

Por todos estos antecedentes, es de interés no sólo para la academia entender la situación socioeconómica de los artesanos macaneros sino también para las autoridades locales con miras a generar procesos de intervención con políticas y acciones que permitan mejorar dichas condiciones y preservar este patrimonio.

\subsection{La Productividad en el Sector Textil Artesanal}

En la última década se han generado múltiples análisis, investigaciones y debates referentes a nuevos conceptos, procesos o formas de repensar en cómo reducir la inequidad, pobreza y marginalidad, es decir; cómo generar desarrollo; entendida ésta desde la mirada del premio Nobel de Economía Amartya Sen "gozar de mayores libertades", es así que se mencionan a la "economía social", "economía popular, "economía solidaria"; en el discurso de muchos gobernantes; en el caso de América Latina, ha surgido como contrapeso al neoliberalismo, que durante su estadía y aplicación de sus recetarios no han generado el ansiado crecimiento económico y peor aún desarrollo de estas economías.

Aparece esta nueva propuesta, en un momento estratégico para avanzar en una economía más justa y solidaria, más humana, más colectiva y por tanto más social; así lo expresa (Coraggio, 2011) al sostener.

El desarrollo de la vida de las personas y comunidades es favorecido por la acción colectiva((...) en donde los conflictos de intereses y la competencia se regula de manera transparente en el seno de la sociedad, donde las relaciones interpersonales fraternales pueden afianzarse sobre vínculos productivos y reproductivos de cooperación generando asociaciones libres de trabajadores antes que empresas (...) lo local lo cotidiano permitirán superar la alienación que implica la concentración de poder en el Estado seccional (p.45)
Estas estructuras asociativas u organizaciones, no deben mirar con estupor el ser llamadas empresas muy distantes de las del enfoque capitalista; sino "empresas con rostro social"; con procesos de participación a rajatabla democráticos en su representación y control de responsabilidades. También hay que estar atentos a la difusión de estas prácticas en el territorio, para que las relaciones establecidas en el mercado, vayan cada vez más generando intercambios con un concepto solidario y de precio justo, para ello se requiere que una parte de su beneficio sea destinado a expandir el sector u otros sectores que estén alineados en la misma dirección.

Esta concepción ha tenido algunos aportes en el transcurso de su existencia, así se puede citar a (Chaves y Monzón, s.f.) qué define a la Economía Social:

"al conjunto de empresas privadas creadas para satisfacer las necesidades de sus socios a través del mercado, produciendo bienes y servicios, asegurando o financiando y en las que la distribución del beneficio y la toma de decisiones no están ligadas directamente con el capital aportado por cada socio, correspondiendo un voto a cada uno de ellos. La Economía Social también incluye a las instituciones sin fines de lucro que son productores no de mercado privados, no controlados por las administraciones públicas y que producen servicios no destinados a la venta para determinados grupos de hogares, procediendo sus recursos principales de contribuciones voluntarias efectuadas por los hogares en su calidad de consumidores, de pagos de las administraciones públicas y de rentas de la propiedad" (p.6).

Partiendo de las concepciones básicas de la economía, los bienes y servicios producidos son un medio para satisfacer las necesidades humanas. Bajo los conceptos de economía y las respuestas que se otorgan a las preguntas del ¿qué, cómo y para quién?, y específicamente el análisis del ¿cómo producir?, autores como (Parkin y Loría, 2010) mencionan que "los bienes y servicios se producen usando recursos productivos, que los economistas llaman factores de producción" (p.3), los mismos que consisten en capital, tierra, trabajo y habilidades empresariales. El capital hace referencia a sus agregados como herramientas, maquinaria, infraestructura, vale decir, los bienes durables, que las empresas utilizan para producir bienes y servicios; en tanto que la tierra comprende todos los recursos naturales que pueden ser utilizados en el proceso productivo. El trabajo según (Parkin y Loría, 2010) es el "tiempo y esfuerzo que la gente dedica a producir bienes y servicios" (p. 4), en donde el tiempo es el número de horas utilizadas por el hombre para desarrollar sus tareas asignadas en un proceso productivo.

La calidad del trabajo está supeditada al capital humano, pues el conocimiento y las habilidades que la gente entrega en el proceso productivo, depende de la educación y la capacitación adquirida en el trabajo y de su trayectoria 
como experiencia laboral, lo que se entendería que mientras más tiempo dedica una persona a la realización de una misma tarea, más conocimiento y habilidad adquiere. Sumado a lo anterior, según Parkín \& Loría (2010), se consideran las habilidades empresariales (Entrepreneurship), que se trata del mismo recurso humano, como el organizador de los tres factores productivos analizados, es decir, la capacidad de gestión del empresario para combinar los distintos factores de producción desde el momento mismo que se asumió el riesgo. Estas actividades serían la dirección, organización, sistematización y conducción para llevar a cabo el proceso productivo combinado.

Hablar de productividad es entender cómo se da la relación entre la cantidad producida y los insumos o medios utilizados que han sido necesarios para alcanzar los beneficios, de entender cómo mejorar la productividad, le va a permitir a la organización responder eficientemente a los cambios globales, generar estrategias que permitan mejorar la productividad ante un entorno cambiante, con recursos financieros y productivos escasos, seleccionar aquellos insumos y métodos que respondan a las necesidades de la empresa y de la realidad local, para ofertar un producto de calidad y precio justo.

La combinación eficiente de estos recursos por medio del trabajo es el que genera el mayor ingreso productivo, así como el aprendizaje práctico, término utilizado por ((Parkin y Loría, 2010), referido a que la repetición de tareas, provoca la elaboración de mayor unidades de bienes producidos, señalando que: "El aprendizaje práctico es un motor poderoso del crecimiento de la productividad, y la ventaja comparativa evoluciona y cambia gracias a la experiencia en el trabajo" (Parkin y Loría, 2010, p.165), es así como el conocimiento aplicado a la producción será obviamente el motor para aumentar la productividad y la competitividad de las empresas.

Por lo tanto, el rol y el alcance que desempeña el capital humano es muy amplio, justamente por la acumulación de conocimiento y habilidad, con repercusiones que trascienden en la dinámica productiva.

\subsection{Factores Determinantes de Productividad}

Algunos autores han desarrollado importantes aportes en el análisis de la competitividad y productividad de empresas y organizaciones locales que les permita atender las demandas de un mercado cada vez más exigente, competitivo y globalizado tales como:

Tabla 1 Factores determinantes de la productividad

\begin{tabular}{|l|l|}
\hline \multicolumn{1}{|c|}{ Autores } & $\begin{array}{l}\text { Factores Determinantes de la Productividad y Com- } \\
\text { petitividad de Empresas Locales }\end{array}$ \\
\hline $\begin{array}{l}\text { (Vázquez Baquero, } \\
\text { 2005) }\end{array}$ & $\begin{array}{l}\text { Plantea que el elemento que impulsa el desarrollo de } \\
\text { un territorio y sus empresas son los procesos de orga- } \\
\text { nización de los sistemas productivos lo que influye di- } \\
\text { rectamente con la productividad, crecimiento y cambio } \\
\text { estructural de las economías. (p.47). }\end{array}$ \\
\hline
\end{tabular}

Tabla 1 Factores determinantes de la productividad...(continuación)

\begin{tabular}{|l|l|}
\hline \multicolumn{1}{|c|}{ Autores } & $\begin{array}{l}\text { Factores Determinantes de la Productividad y Com- } \\
\text { petitividad de Empresas Locales }\end{array}$ \\
\hline (Alburquerque, & $\begin{array}{l}\text { Plantea que las actividades locales para ser competi- } \\
\text { tivas se consideren: la especialización que le permita } \\
\text { tener una ventaja de costos, el liderazgo innovador y } \\
\text { la cooperación que permita aprovechar la experiencia o } \\
\text { especialización en los clústeres territoriales además de, } \\
\text { las externalidades positivas que impulsan la cohesión. } \\
\text { (p.30) }\end{array}$ \\
\hline $\begin{array}{l}\text { (Vázquez Baquero, } \\
1999)\end{array}$ & $\begin{array}{l}\text { Sostiene que son factores determinantes para empresas } \\
\text { locales: la organización de la producción mediante re- } \\
\text { des, la tecnología disponible, la capacidad empresarial, } \\
\text { de la organización y formas de organización social y } \\
\text { sistema de valores. (p: 32-34) }\end{array}$ \\
\hline (Cordero y Otros, & $\begin{array}{l}\text { Señalan como factores a considerar para la mejora } \\
\text { de la competitividad y productividad: Disponibilidad } \\
\text { y calidad de los factores, recursos humanos, recursos } \\
\text { naturales, capital e infraestructura, el entorno y dentro } \\
\text { de este aspecto: marco institucional, demanda interna, } \\
\text { sectores de apoyo, organización social y sistema de } \\
\text { valores. (p:13-16) }\end{array}$ \\
\hline $\begin{array}{l}\text { Concuerda con algunos autores que han abordado la } \\
\text { temática y consideran como factores en el análisis de la } \\
\text { competitividad y productividad: la organización social, } \\
\text { la generación de eslabonamientos, el aprovechamientos } \\
\text { Ge los recursos locales, la infraestructura de apoyo, el } \\
\text { mercado, el ámbito político administrativo y la cultura } \\
\text { local. }\end{array}$ \\
\hline
\end{tabular}

Fuente: Revisión bibliográfica y bases de datos científicas Elaborado: Autoras 2018

Según la OCDE (1950) citado por (Medina, 2009) define a la productividad como “... el cociente que se obtiene de dividir el monto de lo producido entre algunos de los factores de producción"; y estos factores pueden ser el capital, la inversión o las materias primas. Otros autores con el devenir de los años y nuevos insumos que se han ido desarrollando, han permitido ampliar el análisis de productividad en el ámbito empresarial, en términos de: productividad parcial, cuando se hace referencia a un solo insumo; productividad total, cuando se relaciona el resultado total y la suma de todos los factores de la producción; finalmente introduce el parámetro "Índice de la Productividad Total Global" en la que considera como variables determinantes de la productividad "factores intangibles", que engloba aspectos como la "calidad del producto y la calidad de los procesos".

(Smith, 2002) establece el "Principio de la división del trabajo" como mecanismo que permite aumentar la productividad y la rentabilidad. (Taylor, 1973), plantea que para mejorar la productividad se debe analizar científicamente el trabajo y desarrollar mejores métodos para las actividades industriales, esto implica procesos de estandarización en la producción lo que plantea un análisis de productividad parcial porque se centra en medir la productividad de la cantidad de mano de obra utilizada. Así también luego de la segunda guerra mundial se propone desarrollar un Estado de Bienestar, es decir, impulsar desde el Estado políticas que atiendan las demandas sociales e impulsar la productividad para atender las demandas sociales crecientes. A partir de entonces se fueron generando una serie de propuestas y estrategias que impulsen una mayor 
productividad y respondan a procesos de competitividad y globalización de los países.

Para medir la productividad total según Sumath (1990) citado por (Jiménez, Delgado, y Gaona, 2001); “es la razón entre la productividad total y la suma de todos los factores e insumo" (p. 82), esto permite medir la influencia de los factores en la producción. También el autor plantea que para medir una productividad parcial y determinar el impacto de cada uno de los factores productivos, se debe determinar la razón entre la producción y un insumo.

Una teoría también considerada como fundamental en el análisis de la productividad en las Unidades Económicas Domésticas (UED) u organizaciones de las Economía Popular y Solidaria (EPS), se tiene a (Prokopenco, 1989), quien aborda los aspectos de la productividad como factores determinantes en la mejora de la "Justa distribución de la riqueza" (p. 33). Este elemento determinante planteado por el autor se reafirma además al sostener que "las empresas más productivas y las políticas gubernamentales orientadas a la productividad están ligadas a la promoción de una mejor calidad de vida laboral, la participación, los principios económicos, la creatividad, la iniciativa individual y los estilos y prácticas gerenciales" (p. 33). Este autor considera como cualidades y vínculos importantes aspectos como: la competitividad que según lo propuesto por la OCDE citado por (Prokopenco, 1998), a "el grado en que un país puede, bajo condiciones de mercado libre y justo, producir productos y servicios que pasan el test de los mercados internacionales, mientras que simultáneamente mantienen y expanden los ingresos reales de su pueblo en el largo plazo"; la sustentabilidad es decir entender el "cómo generar crecimiento" considerando el menor impacto ambiental; globalización es decir apertura y no marginalización, impulsar alianzas y cooperación que permita alcanzar mayor competitividad, y; calidad del recurso humano y la competencia gerencial con un enfoque de "catalizador proactivo para el cambio" que promueva "conductas productivas, prácticas gerenciales humanistas y comunicación abierta" (p. 34-62).

Según (Prokopenco, 1989) define a la productividad "como el uso eficiente de recursos -trabajo, capital, tierra, materiales, energía, información - en la producción de diversos bienes y servicios" (p. 3)

La OCDE en un análisis de las condiciones de productividad para América Latina, plantea que un reto importante de la región es conciliar dos elementos básicos de la economía, como son: reducir las brechas de productividad y la inclusión social, que no se han logrado superar debido a factores estructurales; por ello, los gobiernos cumplen un rol determinante en la consecución de mejoras en la productividad, a través de la generación de políticas que permitan procesos de mayor inclusión y mejor distribución de los ingresos, aprovechamiento de las competencias de la población, mayores niveles de educación, impulsar y potenciar desde el estado el desarrollo de las pymes, dentro de un marco legal que las promueven, para que no sólo se constituyan dentro de la informalidad por las excesiva carga tributarias que contrae su crecimiento y no permite que operen en mercados internacionales accediendo a procesos de innovación, competencias a través de mejoras de la educación y promoción de empleos de calidad, acceso a recursos e infraestructura (Económico, 2016)

La teoría neoclásica plantea la productividad considerando diversos factores como determinantes de la misma, por ello se plantea que la productividad depende de una multiplicidad de factores, pero como no todos son medibles para ello se propone la teoría de Solow (1957) citado por (Valle, 1991), quien propone la "productividad multifactorial". Mientras que la teoría marxista plantea que "la productividad es el recíproco del valor, del trabajo abstracto socialmente necesario para la producción de las distintas mercancías"; este trabajo abstracto entendido como "cada mercancía es en rigor el resultado de la aplicación de una porción del trabajo social y no sólo del proceso de trabajo que aparece inmediatamente como su origen" (p. 9).

(Medina, 2009)), plantea que "el nivel de producción debe estar relacionado con los insumos que son necesarios para producirlo. Mientras aumente la relación produccióninsumos, se obtiene una productividad más alta" (p.28). Así mismo Kotler (2009) citado por (Asencio y Neira, 2018), plantea con relación a las pequeñas y medianas empresas manufactureras que por tener "una estructura manejable, tienen potencial para responder rápidamente a oportunidades de negocios porque no tienen una organización complicada (...), a la vez pueden tener cuidadoso control de su crecimiento, lo que les facilita penetrar nuevos mercados" (p. 358).

En tanto que para analizar la productividad del sector artesanal es necesario partir de entender el sistema productivo artesanal y sus características, tomando la definición de la (Organización de las Naciones Unidas para la Educación, 1997); sostiene que la producción artesanal puede tener 3 sistemas de producción: Totalmente manual o artesanal, mixto-manual y con ayuda de herramientas manuales, y mecánicas, pero siempre con predominio de la actividad manual del artesano, utilizan materia primas locales, y sus productos se basan en características distintivas vinculadas a la cultura propia de la zona y de la región.

Otros aspectos a destacar en el análisis de la actividad artesanal y de la importancia que representa para el territorio es su sostenibilidad, que según un análisis de experiencias en este sector, levantado en 11 países de la región por (Dini, Ferraro, y Gasaly, 2007), plantean que se deben generar políticas que promuevan la "innovación y el acceso a mercados más exigentes", pero para lograr estos procesos y mejorar la productividad y la inserción al mercado global, concluyen que es necesarios generar alianzas o contacto directo con los clientes que permita una transferencia de conocimientos e información necesaria para el productor, para introducir procesos de innovación técnica y satisfacción de la demanda, así como para acceder a mercados internacionales es necesario: "estandarizar pro- 
cesos productivos, incorporar estándares de calidad, controles, reducir tiempos de entrega , mejorar el control de las ventas y potenciar su capacidad logística" citado por (Ortiz y López, 2015).

(Vega, 2012) sostiene en el análisis de los aspectos productivos, comerciales y organizacionales de las actividades artesanales, que estas poseen un valor adicional a otras actividades, por cuanto permiten precautelar la historia y conservar las tradiciones de los pueblos, pero estos elementos según el análisis propuesto no son una garantía para mejorar las condiciones de vida de los artesanos y por ende mejores ingresos, es por ello que si los gobiernos locales buscan preservar los saberes ancestrales, es necesario desarrollar políticas y esfuerzos que permitan mejorar las condiciones de vida de la población dedicada a la actividad artesanal.

\subsection{Aspectos Culturales e Identitarios de la Actividad Artesanal}

En los procesos productivos actuales de las organizaciones según (Ríos, 2018), las empresas buscan sostenerse en el mercado y para ello es importante fortalecer el liderazgo, la innovación y la productividad es decir, la "Teoría de la ventaja competitiva" de Ricardo en 1817 , planteaba que el país o región que tenga menores costos en sus procesos puede beneficiarse del comercio; esto también se puede aplicar a las actividades artesanales dado que la experiencia y experticia que poseen en las técnicas de producción le infunden un valor agregado permitiendo una mayor producción y por ende una mejora en los ingresos y calidad de vida.

Ramírez 1986 citado por (Ramón, Ramírez, Navarro, y Estrella, 2008), sostiene que las artesanías son aspectos culturales que identifican al territorio, así también (García, 1990), propone que aplicando lógicas de red y de articulación interterritoriales el desarrollo que ha tenido esta actividad y su presencia en los mercados ha sido producto de la valoración y reconocimiento que se le ha dado a sus características simbólicas y además por sus mejoras tecnológicas e innovación. Otros elementos a destacar de la productividad del sector textil artesanal según (Novelo, 1976), son las relaciones sociales que caracterizan a la actividad, además destaca que la actividad se caracteriza por ser de tipo manual y de tipo familiar.

(Aurier, Fort, y Sirieix, 2005), plantean que el elemento de "territorio" a los productos por ser propios del mismo, le ha permitido generar una imagen de diferente o exclusivo de la región o de la localidad lo que le imprime al producto una percepción de calidad; este hecho ha generado para el caso de las actividades artesanales manuales que se llevan a cabo en diferentes zonas ser consideradas de calidad única e identitaria, logrando posicionarlas y volverlas atractivas para el mercado; este elemento les permite mantenerse en el mercado y competir con actividades similares pero de tipo industrial, porque poseen características especiales por su propio proceso productivo manual. Los autores establecen tres factores que lo diferencian al producto: “1) la referencia al lugar, 2) la asociación con el tiempo y la cultura y, 3) la aceptación de un saber hacer" (p. 985)

\section{Metodología}

La línea de investigación en la que se enmarca este trabajo es la de economía social y solidaria, con enfoque cuantitativo de tipo no experimental - transversal y descriptivo, porque analiza la relación entre las variables de producción, ingresos, edad de los artesanos y el tiempo de dedicación a la actividad en un momento dado, sin manipulación intencional de las variables, el fenómeno ha sido considerado tal como se ha presentado en el contexto.

Para la fundamentación teórica se ha utilizado la técnica de revisión de bases científicas y bibliografía relevante; entre los autores más destacados están: Prokopenko, Sumanth, Parkin \& Loría, OCDE, Smith, Taylor, Vázquez Baquero, Cordero y otros, Medina, entre otros.

El universo de estudio se centró en 36 artesanos de macanas ubicados en la zona de Bullcay y Bulzhún; con los datos obtenidos en la encuesta del proyecto de vinculación realizado por la carrera de Economía de la Universidad Católica de Cuenca y el GAD cantonal de Gualaceo en el año 2016, se determinó el índice de productividad parcial del sector y la correlación entre los ingresos, edad y cantidad de producción, además de los estadísticos descriptivos, cálculos y gráficos con la ayuda de los procesadores de información IBM SPSS statistics 2.0 y hoja de cálculo de Excel.

En primera instancia se obtuvieron los estadísticos descriptivos generales del sector en donde se establece la media y desviación típica de edad, unidades producidas e ingresos de los artesanos; luego se organizó la edad de los mismos estableciendo rangos, en los cuales se muestran la media de la cantidad de producción e ingresos por cada uno.

Posteriormente se establece dos coeficientes de correlación, el primero entre la edad y la cantidad de producción y el segundo entre la edad y los ingresos de cada artesano.

Para el cálculo del índice de productividad se consideró el postulado de Sumanth y la OCDE, que propone dividir el total de la producción para uno o algunos de los factores de la producción, por el ello se dividió la cantidad de producción total mensual del sector para la cantidad total de horas trabajadas en el mes, considerando como base la jornada laboral vigente en el Ecuador.

\section{Resultados}

Los resultados que se exponen a continuación parten desde los objetivos propuestos inicialmente y mantienen relación con el problema de investigación, la metodología aplicada y la base teórica considerada.

En la tabla 2 se muestra que en promedio la edad de los artesanos es 57 años, elaboran 7 macanas al mes, lo que equivale a ingresos de 143.67 USD. 
Tabla 2 Estadísticos descriptivos 1

\begin{tabular}{|l|c|c|c|c|c|}
\hline & N & Mínimo & Máximo & Media & Desv. típ. \\
\hline Edad del encuestado & 36 & 32 & 85 & 57,39 & 13,462 \\
Ingresos & 36 & 30,00 & 1350,00 & 143,6667 & 222,82511 \\
Cantidad de macanas producidas & 36 & 2 & 60 & 6,86 & 9,772 \\
N válido (según lista) & 36 & & & & \\
\hline
\end{tabular}

Fuente: Encuesta del Proyecto de Vinculación

Elaborado: Autoras 2018

Tabla 3 Estadísticos descriptivos 2

\begin{tabular}{|c|c|c|c|c|c|c|c|}
\hline \multicolumn{2}{|l|}{ Rangos de edad } & $\begin{array}{c}\text { No. } \\
\text { Frecuencias }\end{array}$ & $\%$ & $\begin{array}{c}\text { Q. } \\
\text { PR OD. } \\
(\mathrm{Q} .)\end{array}$ & $\overline{\mathrm{O}}$ & $\begin{array}{c}\text { INGRESOS } \\
(\mathrm{I})\end{array}$ & $\overline{\mathrm{I}}$ \\
\hline 32 & 41 & 5 & 13.9 & 26 & 5 & 655 & 131 \\
\hline 42 & 51 & 7 & 19.4 & 93 & 13 & 1952 & 279 \\
\hline 52 & 61 & 12 & 33.3 & 81 & 7 & 1586 & 132 \\
\hline 62 & 71 & 6 & 16.7 & 20 & 3 & 356 & 59 \\
\hline 72 & 81 & 4 & 11.1 & 19 & 5 & 460 & 115 \\
\hline 82 & 91 & 2 & 5.6 & 8 & 4 & 163 & 82 \\
\hline
\end{tabular}

Fuente: Encuesta del Proyecto de Vinculación

Elaborado: Autoras 2018

En la tabla 3 se aprecia que los rangos de edad con mayor producción de unidades de macanas e ingresos al mes se encuentran entre los 42 y 61 años, de los cuales el rango que va de 42 a 51 años representan el $19.4 \%$ del total de artesanos en esta actividad con una media de producción e ingresos de 13 unidades y 279 USD, respectivamente. En tanto que los que se ubican entre los 52 y 61 años de edad representan el $33.3 \%$ de la población artesanal, con medias de 7 unidades de producción e ingresos 132 USD al mes.

Se puede ver también que los artesanos ubicados en los rangos de edad de 32 a 41 años presentan la misma media de 5 unidades producidas que los que se encuentran en un rango de 72 a 81 años, sin embargo; los ingresos medios varían de 131 USD a 115 USD respectivamente.

Figura 1. Correlación entre Edad y cantidad de unidades producidas

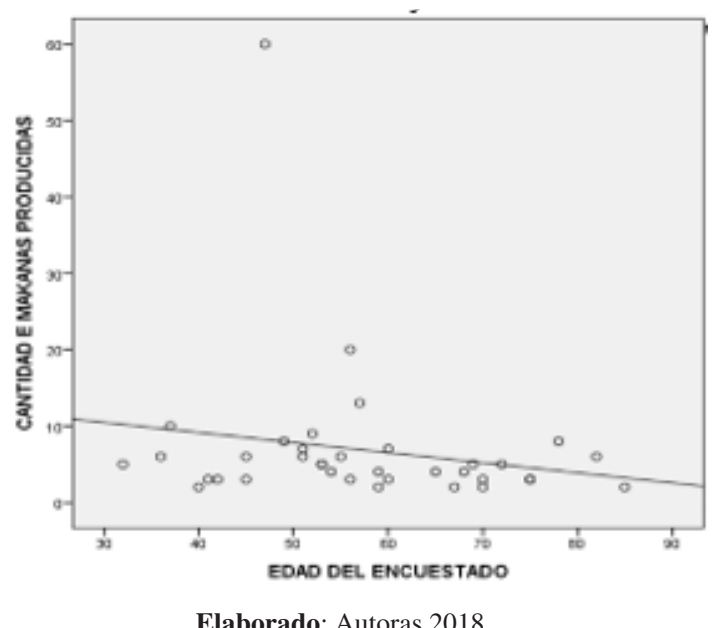

La figura 1 presenta la relación entre la edad de los artesanos y la cantidad de unidades de macanas que producen al mes, lo que da como resultado un índice correlacional de $\rho=-0,182$, interpretándose como la fuerza y asociación lineal entre las variables muy débil e inversamente proporcional.

Figura 2. Correlación entre Edad e Ingresos

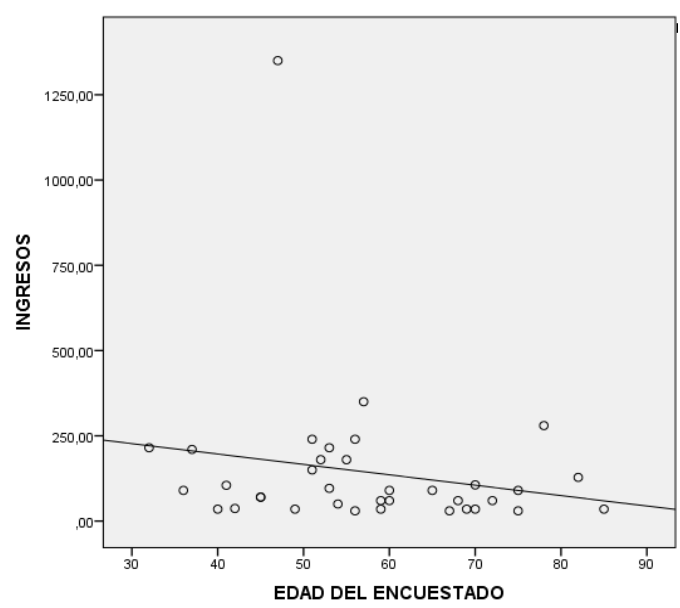

Elaborado: Autoras 2018

En la figura 2 se muestra la correlación entre la edad de los artesanos y los ingresos que perciben, cuyo índice da como resultado $\rho=-0,184$; lo que significa que la fuerza y asociación lineal entre las variables es muy débil e inversamente proporcional.

\subsection{Cálculo de Índice de Productividad Parcial}

$$
\begin{aligned}
\text { P.p. } & =\frac{P . T .}{t} \\
\text { P.p. } & =\frac{247 u d .}{5760 h} \\
\text { P.p. } & =0,043 \text { unid./hora }
\end{aligned}
$$

P.p. = producción parcial

P.T. = producción total de unidades producidas

$\mathbf{I}=$ Ingresos

$\mathbf{t}=$ tiempo expresado en horas

Las 247 unidades consideradas corresponden a la producción total mensual de macanas del sector, este valor es dividido para 5760 horas de trabajo al mes, que resulta de la multiplicación de la jornada laboral que rige en el 
Ecuador establecida en 160 horas mensuales, por el total de artesanos del sector.

El índice de productividad es de 0,043 ud./hora, lo que equivale a la producción de 7 macanas por mes que, al ser multiplicado por el precio promedio de venta, el artesano estaría generando un ingreso de 238 USD mensuales, que al ser comparado con un Salario Mínimo Vital (SMV) de 386 USD, se observa un diferencial de 148 USD.

\section{Conclusiones y Recomendaciones}

Los factores de productividad que inciden en los ingresos del sector artesanal son la disponibilidad y calidad de recursos humanos, naturales, capital e infraestructura, el entorno, marco institucional, demanda interna, sectores de apoyo y cultura local.

La relación entre las variables de productividad e ingresos asociadas a la edad en el sector artesanal de macanas con base en el resultado obtenido en el índice de Pearson es muy débil e inversamente proporcional.

La edad no determina la cantidad de unidades producidas por artesano, la edad promedio de los artesanos bordea los 57 años y en promedio elaboran 7 unidades con ingresos de 143.67USD. Si bien la población de mayor edad tiene más experticia produce menos, y los que se encuentran en un rango menor de edad producen más, pero representan a un grupo menor de la población. Aproximadamente el $67 \%$ de los artesanos no se dedican $100 \%$ a la actividad y complementan sus ingresos con otras actividades, esto lleva a que la productividad del sector sea baja.

El índice de productividad parcial del sector macanero de las parroquias de Bullcay y Bullzhún es bajo, al generar ingresos por debajo de un SMV. Para alcanzar un SMV de 386 USD, el nivel de productividad debería ser de 0.075 ud./hora, que equivale a una producción de 431 macanas, significando que los macaneros deberían incrementar en 5 unidades su producción mensual.

El índice de productividad obtenido refleja la realidad del sector que desarrolla una actividad artesanal manual que demanda mayor tiempo de dedicación y no existe el reconocimiento al valor intangible como conocimiento y cultura, en términos monetarios en el mercado.

La situación socioeconómica de los artesanos macaneros es similar a la de otros a nivel mundial, pese a tener una gran riqueza cultural están supeditados al mercado y esto ha hecho que las familias artesanas vivan en condiciones de pobreza; por ello la población joven no ve esta actividad como una alternativa viable de supervivencia, ya que por un lado ven el esfuerzo permanente de sus mayores dedicando más de 8 horas a la actividad y su retribución no representa el valor del trabajo incorporado en el producto final, por esta razón los artesanos son de edad avanzada.

\section{Referencias Bibliográficas}

Alburquerque, F. (1999). Desarrollo Económico Local en Europa y América Latina. Santiago de Chile:
Instituto Latinoamericano de Planeación Económica y Social (ILPES).

Amoroso, G., Calle, M., Campoverde, R., y Rosales, M. (2018). Análisis Correlacional entre el Nivel de Ingresos y los Canales de Comercialización de los Artesanos del Cantón Gualaceo que Utilizan la Técnica Ikat. Revista XIII Congreso de Ciencia y Tecnología. ESPE.

Asencio, L., y Neira, G. (2018). Análisis de factores de competitividad y su incidencia en la gestión de ventas del mercado. Publicando, 352-369.

Aurier, P., Fort, F., y Sirieix, L. (2005). Explorando los significados del producto trroir para el consumidor. Descargado de http: / / https / / journals . opendition.org/aof/187

Chaves, R., y Monzón, J. (s.f.). Economía Social y Sector no Lucrativo: Actualidad Científica y Perspectivas. CIRIEC, 7-33.

Cordero, P., y Otros, y. (2003). "Territorios Rurales, Competitividad y Desarrollo", en Cuaderno Técnico $N^{o}$ 23. San José, Costa Rica: IICA.

Díaz, E., Sánchez, R., y Guadarrama, D. (2006). La actividad Textil de Guadalajara Yancuictlalpan ¿un sistema productivo local? Quivera, 210-230.

Dini, M., Ferraro, C., y Gasaly, C. (2007). Pymes y articulación productiva. REsultados y lecciones a partir de experiencias en América Latina. Serie desarrollo productivo.

Económico, O. p. 1. C. y. e. D. (2016). Fomentando un crecimiento inclusivo de la Productividad en América Latina. Descargado de http: //www. oecd - org/about/publishing/

García, N. (1990). Culturas híbridas. Estrategias para entrar y salir de la modernidad. México: Gijalbo.

Jiménez, A., Delgado, E., y Gaona, G. (2001). Modelo de productividad de David Sumanth aplicado a una empresa del sector de maquinaria no eléctrica. Academia y Desarrollo, 81-87.

Medina, J. (2009). Modelo Integral de Productividad. Una Visión Estratégica. Bogotá: Digitprint editores.

Mordo, C. (2002). Revista Artesanías de América. Descargado de http://documentacion.cidap . gob.ec:8080/handle/cidap/1465

Organización de las Naciones Unidas para la Educación, 1. C. y. 1. C. U. (1997). Simposio UNESCO/CCI. Manila.

Ortiz, M., y López, R. (2015). Caracterización socioeconómica de la comunidad artesanal de Nariño, Colombia. Lecturas de Economía, 247-281.

Parkin, M., y Loría, E. (2010). Microeconomía Versión para Latinoamérica. México: Pearson.

Prokopenco, J. (1989). La Gestión de la Productividad. Ginebra: Oficina Internacional del Trabajo.

Prokopenco, J. (1998). Globalización, competitividad y estrategias de productividad. Boletín Cinterfor, 3370 . 
Ramón, D., Ramírez, J., Navarro, H., y Estrella, N. (2008). Etnocompetitividad del sistema artesanal textil Mitla, el papel del territorio y la innovación. $s / n, 981-1006$.

Smith, A. (2002). La riqueza de las Naciones. Madrid: Alianza.

Taylor, F. (1973). La Administración Científica. Buenos Aires: El Ateneo.

Vázquez Baquero, A. (1999). Desarrollo Redes e Innovación. Lecciones sobre el Desarrollo Endógeno. Madrid: Ediciones Pirámide, S.A.

Vázquez Baquero, A. (2005). Las Nuevas Fuerzas del Desarrollo. Barcelona: Antonio Bosh Editor S.A.

Vega, D. (2012). El aprendizaje de la Artesanía y su Reproducción Social en Colombia. Educación y Territorio, 2(1), 89-112. 
